\title{
A General Perspective of Microbiota in Human Health and Disease
}

\author{
Mbuvi P Mutua ${ }^{1 *}$, Shadrack Muya ${ }^{2}$ and Gicheru M Muita ${ }^{1}$ \\ ${ }^{1}$ Department of Zoological Sciences, Kenyatta University, P.O Box 43844-00100, Nairobi, Kenya \\ ${ }^{2}$ Department of Zoology, Jomo Kenyatta University of Agriculture and Technology, P.O Box 62000-00200, Nairobi, Kenya
}

${ }^{*}$ Corresponding author: Mbuvi P Mutua, Department of Zoological Sciences, Kenyatta University, P.O Box 43844-00100, Nairobi, Kenya, Tel: 254727677738; E-mail: patmbuvi@gamil.com

Received date: February 03, 2020; Accepted date: February 18, 2020; Published date: February 27, 2020

Citation: Mutua MP, Muya S, Muita G (2020) A General Perspective of microbiota in Human Health and Disease. Arch Clin Microbiol Vol. 11 No. 2:106

Copyright: (C) 2020 Mutua MP, et al. This is an open-access article distributed under the terms of the Creative Commons Attribution License, which permits unrestricted use, distribution, and reproduction in any medium, provided the original author and source are credited.

\section{Abstract}

The study of human microbiome is widely perceived to be a young biomedical discipline. Recent studies indicate an association between human microbiome and chronic disease conditions such as diabetes and obesity. An understanding of human microbiome structure and function is important for design and delivery of microbial based therapies especially against immunological and metabolic chronic diseases. In this review, we demonstrate the role human microbiota in health and disease in various anatomic sites and in development of neonate immunity. In particular, the review focuses on the role of placental microbiota in fetoplacental unit receptivity and the effect of early microbiota exposure on neonate immunity development. Additionaly, microbiota impact on health and disease in the gut, lung and skin is explored.

Keywords: Microbiota; Neonate; Dysbiosis; Pregnancy; Lungs; Skin; Gastrointestinal tract

\section{Abbreviations:}

AMP: Antimicrobial Peptides; IFN $\beta$ : Interferon Beta

\section{Introduction}

Dysbiosis refers to compositional and functional changes of the microbiome and it can result into changes of all or any of the following microbiome characteristics: Microbiome stability, Microbiome diversity, and Microbiome resilience [1]. Microbiome stability also referred to as microbiome resistance is the amenability of microbiota to perturbations. Microbiome diversity refers to richness of the microbiota ecosystem. Microbiome resilience is the ability of microbiota to get restored to pre-perturbation state. Dysbiosis can be driven by environmental and host related factors. However, variability of microbiota among healthy individuals of different age, geographical limits and dietary habits, limits the definition of what actually constitutes the dysbiotic state. In view of this limitation, [1] have defined dysbiosis as a microbial community state that is not only statistically associated with disease, but also functionally contributes to etiology, diagnosis, or treatment of the disease.

\section{Literature Review}

Dysbiotic state can fall in either of the following categories: (a) Decline of commensals which is either a reduction or complete loss of microbiota and can be caused by either direct killing of microbiota or attenuation of microbiota proliferation [2]. Loss of commensals has been observed in Clostridium difficile induced inflammation and restoration of the diminished Clostridium scindens has been reported to ameliorate the inflammatory condition [3]. (b) Dysbiosis can also result from growth of commensal microbiota that has potential to cause pathology; such commensals have been referred to as pathobionts [4]. Studies report existence of pathobionts at low relative abundance, but grow when there is impairment of the microbial ecosystem. This type of dysbiosis has been observed in the Entero bacteriaceae, whose bloom is commonly observed in enteric inflammation [3]. (c) Loss of microbiota species diversity within a site, known as decline in alpha diversity, also constitutes dysbiosis and this type of microbiota perturbation has been linked to metabolic health [5]. Alpha diversity in the intestinal microbiota increases during the first years of life and is a function of dietary patterns [6]. Low intestinal bacterial diversity has been documented in AIDS, Intestinal bowel disease and type 1 diabetes [7], and this has been attributed to abnormal dietary composition [8].

\section{Microbiota in pregnancy}

The role of trophoblast cells in regulating immune activities at the maternal-fetal interface is well documented. Trophoblast cells can promote a tolerogenic phenotype, sense and respond to pathogen associated molecular patterns present in microorganisms, and that a breach of the trophoblast immunity can cause pregnancy complications 
including preterm birth [8,9]. Bacterial infections account for more than $40 \%$ of preterm birth cases [10]. Infectious bacteria can access maternal-fetal interface by descending from peritoneal cavity, from maternal circulation or by ascending from the lower reproductive tract [11]. Research findings indicate that bacterial infections at the maternal-fetal interface weaken the trophoblast capacity to induce and promote fetal acceptance and, instead upregulate trophoblastic inflammatory immune reactions with subsequent fetal loss [12]. Despite strong evidence linking bacterial infections to pregnancy complications, antibiotic treatment has not proven effective [13]. Recent studies demonstrate existence of placental microbiota to play a critical role in success of pregnancy [14]. Escherichia coli and other Proteo bacteria family, have been shown to be abundant placental microbiome $[15,16]$. It is now documented that placental microbiota activities induce tolerogenic immunity, thereby permeating receptability and preventing rejection of fetal-placental unit [17]. Additionally, exposure of the fetus to maternal microbiota during pregnancy can significantly affect development of postnatal immunity in the neonate [18]. Maternal-fetal interface microbiota improves trophoblast expression of IFN $\beta$. IFN $\beta$ modulate maternal immune system with increased maternal-fetal tolerance and receptivity [19]. IFN $\beta$ belong to type 1 IFNs that trigger programmed cell death in activated T-cells and increase production of immunosuppressive molecules at the maternal-fetal interface [20]. Viruses inhibit type 1 IFN pathway in the trophoblast. Consequently, placental microbiota capability to induce an immunosuppressive, tolerogenic trophoblast type 1 IFN pathway can be abolished by viral infections [21]. Further, viral infections shit placental microbiota milieu that has an immune-tolerant setting to a pro-inflammatory state [22].

\section{Neonate microbiota and disease development}

Early life microbiota affects allergy development later in child hood. Studies indicate low intestinal microbiota diversity during the first month of life is associated with allergic sensitization and asthma in children aged 6-7 years [23]. Colonization with Bifidobacterium breve is associated with reduced risk of atopic dermatitis in the first year of life but Bifidobacterium catenulatum colonization is linked to a higher risk of atopic dermatitis [24]. In a Canadian study of infants, low abundance of Faecalibacteria, Lachnospira, Veillonella and Rothia genera at 3 months following parturition was associated with higher risk of asthma and allergy development [25]. In a study conducted in the US, infants with lower abundance of species within Lactobacillus, Faecalibacteria, Bifidobacterium and Akkermansia genera at 1 month of age had a higher chance of getting asthmatic attack at the age of 4 years compared to those with higher abundance of the genera. The authors of the study suggested that long-term immunological consequences of the specific early life microbiota profiles could be exerted through production of distinct microbiota metabolites [26].

\section{Skin microbiota}

The skin surface is a lipid and protein rich cornified layer, occasionally with invagination interruptions where follicles are located. The skin surface and follicles are both physically and chemically distinct $[27,28]$. The skin microbiota exhibits specific site distribution patterns with Cutibacterium and Staphylococcus predominantly residing in sebaceous areas such the face and torso while Corynebacterium, beta Proteobacteria and Staphylococcus dominating moist surfaces such as the armpits [29]. This microbiota distribution pattern indicates skin niche chemistry drives microbiome distribution [29]. Skin microbiota assembly process begins during birth [30] but the microbiota shifts notably during puberty, with increased predominance of Corynebacterium and Cutibacterium and a decline in abundance of Firmicutes [31]. However, in adulthood, skin microbiota remains stable over time [32].

Skin microbiota modulate the expression of various innate factors such as the components of complement [33] and antimicrobial peptides (AMP) that are majorly in form of cathelicidins and $\beta$-defensins. Cutibacterium stimulate production of AMP in keratinocytes and sebocytes [34] while Staphylococcus epidermidis have been demonstrated to produce AMP $[35,36]$. Corynebacterium microbial members constitute a major skin microbiota genus. Corynebacterium and Mycobacteria genera share common microbiological features such as similar surface and cellular structures. It is not yet clear how the skin immune system distinguishes between bacteria with such similar features [37]. Structurally, Corynebacterium cell wall has lipoglycans termed lipomannas and lipoarabinomannas both of which are ligands for the host Toll-like-receptors and C-type lectins. The binding of the ligands and receptors triggers a pro or anti-inflammatory response depending on the immunological context in which the ligand-receptor complex is sensed [38]). Recent studies report microbe-microbe interactions to impact on human health. The skin microbiota Corynebacterium accolens inhibits growth of Streptococcus pnuemoniae, a common respiratory tract pathogen [39]. This interaction is mediated by corynebacterial lipase which hydrolyses triolein to release oleic acid which in turn inhibits pneumococcal growth [39]. Skin resident Staphylococcus epidermidis produce AMP that kill Staphylococcus aureus and transplantation of Staphylococcus epidermidis into the skin of patients with atopic dermatitis is known to decrease colonization by Staphylococcus aureus [36]. Further, Staphylococcus epidermidis confer cutaneous immune protection against infections by activating keratinocytes to produce AMP [40]. Therefore, skin microbiota immune protection goes beyond competitive exclusion.

\section{Lung microbiota}

Historically, the dogma that lung is a sterile organ has been held, however, in the last decade, studies have demonstrated new knowledge that the lung is not sterile and actually, the organ harbors a diverse interacting microbiota [41,42]. However, there is dearth of information regarding the potential role of lung microbiota in regulation of lung immune 
response and homeostasis. The lung is incessantly exposed to microbiota either through inhalation or subclinical aspiration from birth [43]. According to a study [44], the lung is exposed to 7000 liters of diverse microbe rich air every day. The upper respiratory tract where microbes are found in abundance is in direct communication with the lung and sub clinical aspiration of oropharyngeal microbial rich content occurs frequently in humans $[45,46]$. The respiratory tract mucociliary system ensures a constant level of microbe immigration and elimination in the lung. Using a high-throughput sequencing of amplicons of the 16s rRNA gene, a highly conserved locus in the bacterial genome, a study [47] demonstrated that bacteria in healthy airways were similar but distinct from airways of asthma patients, who predominantly had Proteobacteria phylum. The major bacterial phyla in healthy lung reported by various studies are Bacteroidetes, and Firmicutes [47-49]. There is relatively low bacterial biomass in the human lung. Human bronchoalveolar lavage studies have reported biomass from 4.5 to 8.25 log copies/Ml [50,51]. Newborns acquire skin microbiota that resembles their mothers' microbiome in a manner that is specific to the method of delivery. Infants born by vaginal route acquire skin bacterial communities resembling mothers' vaginal microbiota dominated by Lactobacillus, Sneathia or Prevotella, while those born by cesarean section acquire microbiota resembling that of mothers' skin microbiota composed of Corynebacterium, Cutibacterium and Staphylococcus species [52]. However, studies that have examined the dynamic changes that may occur in the lower respiratory tract microbiota during pregnancy and as childhood progresses are scarce. In the lungs, conditions that affect bacterial proliferation include oxygen tension, local $\mathrm{pH}$, epithelial cells structure, blood flow and effector inflammatory cell profile [53,54]. Further chronic lung diseases may considerably alter microbial community through elevated volumes of micro aspiration achieved by cough and mucociliary clearance [55]. A study [56], for instance, reported increase in abundance of Prevotella and Veillonella in lymphocyte and neutrophil mediated lung inflammation. Chronic lung diseases are characterized by interludes of acute exacerbations marked by decline in pulmonary function and worsening pulmonary symptoms. Such exacerbations are the main cause of morbidity and mortality. Chronic lung disease exacerbations are indicative of disease progression and many patients fail to restore to their pre-perturbation functional and physiological state, a situation that has been associated with lung microbiota dysbiosis [57]. There is, for example, a decline in abundance of lung Bacteroidetes in asthmatic airways [47]. In another study, lung Proteobacteria were the predominant microbiota found during bronchial hyper-responsiveness [58]. Additionally, host microbiome interaction has been reported as a critical aspect of asthma development. Children born with broad lung microbial exposures were reported to be protected from asthma and atopy [58]. Further, an association between a higher fiber diet and reduced risk of asthma has been demonstrated [59].

\section{Gut microbiota}

The human gut harbors an estimated 1000 bacterial species and that, gut microbiota dysbiosis has been associated with multifactorial disease conditions such as inflammatory bowel disease, obesity and type 2 diabetes interlia [60]. The gut microbiota of a healthy human confers a number of health benefits including pathogen protection, immune modulation and nutrition [61]. Following birth, the neonate gut is rapidly colonized by microbiota and this has been reported to depend on mode of delivery, feeding type and use of antibiotics [62]. Early gut microbial members include enterobacter and enterococci followed by anaerobic Bifidobacteria, clostridia, and Bacteroides species [63]. In the adult gut microbiota, the predominant bacterial phyla reported are Firmicutes and Bacteroidetes. Other phyla present in low abundance include Actinobacteria, Proteobacteria, Fusobacteria and Verrucomicroba [64]. The butyrate producing bacteria Faecalibacterium, Roseburia and Bacteroides have also been identified in a healthy human gut microbiota [65]. It has been documented that there is age related alterations in the gut microbiota of elderly people. In a study of people aged over 65 years, the predominant gut microbiota was found to be Bacteroidetes and this was associated with the low grade inflammation, otherwise referred inflammaging, of the gastrointestinal tract in the elderly [66]. A number of diseases have been linked with gut dysbiotic state. In inflammatory bowel disease which is characterized by chronic and relapsing inflammation of the intestinal tract, gut dysbiosis has been associated with the development of mucosal lesions [67]. Lower abundance of the Bacteroidetes and Firmicutes phyla has been reported in intestinal bowel disease [68]. Additionally, the gut microbiota Roseburia needed for butyrate production and the Phascolarcto bacterium needed for succinate production are significantly low in intestinal bowel disease [69]. Several studies suggest a role of gut microbiota in the pathogenesis of colorectal cancer $[70,71]$. It has been reported that abundance of Fusobacteria to increase in colorectal cancer tumors compared to control samples [71]. In particular, Fusobacterium nuclaetum, Fusobacterium necrophorum and Fusobacterium mortiferum were enriched in tumor tissue but there was decline in proportions of Firmicutes and Bacteroidetes [71]. It is thought that Fusobacterium contribute to colorectal cancer pathogenesis by eliciting tumor inflammation [72]. Irritable bowel syndrome, marked by abdominal pain and altered bowel habits, gut dysbiosis has been associated with the disease low gradeintestinal inflammation [73]. Further, gut dysbiotic state has been suggested to facilitate pathogen adhesion in irritable bowel syndrome [74]. In irritable bowel syndrome, there is considerable increase in abundance of Firmicutes, Ruminococcus, Clostridium, and Dorea and a decline in proportions of Bifidobacterium and Faecalibacterium species [75]. There is paucity of information regarding association between gut microbiota and obesity. However, a study [60] established a decrease in the ratio of Firmicutes to Bacteroidetes following weight loss in human subjects. In another study, a lower proportion of Bacteroidetes in obese individuals but increased abundance of Actinobacteria with a 
fairly stable proportion of Firmicutes was reported. In type 2 diabetes, which is principally linked with obesity related insulin resistance, there is low proportion of Firmicutes but a higher proportion of Bacteroidetes compared to normal controls. Further, type 2 diabetes patients have a gut dysbiosis with a higher proportion of opportunistic pathogens such as Clostridium species but a lower proportion of butyrate producing bacteria [65].

\section{Conclusion}

The human microbiome plays a critical role in human health and disease. Resident microbiota in different anatomic sites of human body influence metabolism, physiology and modulate immune system development. Perturbation of microbiota is associated with several multifactorial disease conditions and this is fundamental in development of appropriate therapeutic approaches. Targeting of specific constituents of microbiota may potentially permeate removal of harmful microorganisms and or their metabolites and enrichment of beneficial microbes.

\section{Declarations}

\section{Competing interests}

The authors declare that they have no competing interests.

\section{Funding}

We thank Louise Bowditch who provided funds for this review.

\section{Authors' contributions}

MPM wrote the manuscript. MSh and GM provided additional information, read and improved the manuscript.

\section{Acknowledgements}

Not applicable.

\section{Competing interests}

The authors declare that they have no competing interests.

\section{References}

1. Maayan L, Aleksandra A, Kolodziejczyk AA, Thaiss CA, Elinav E (2017) Dysbiosis and the immune system. Nat Rev Immunol 4: 219-232.

2. Krem T, Zeevi D, Suez J, Weinberger A, Avnit-Sagi T, et al. (2015) Growth dynamics of gut microbiota in health and disease inferred from single metagenomics samples. Science 349: 1101-1106.

3. Buffie CG, Bucci V, Stein RR, McKenney PT, Ling L, et al. (2015) Precision microbiome reconstitution restores bile acid mediated resistance to Clostridium difficile. Nature 517:205-208.
4. Stecher B, Maier L, Hardt WD (2013) "Blooming" in the gut: how dysbiosis might contribute to pathogen evolution. Nat Rev Microbiol 11: 277-284.

5. Chow J, Mazmanian SK (2010) A pathobiont of the microbiota balances host colonization and intestinal inflammation. Cell Host Microbe 7: 265-276.

6. Emmanuel LC, Trine N, Junjie Q, Edi P, Falk H, et al. (2013) Richness of human gut microbiome correlates with metabolic markers. Nature 500: 541-546.

7. Kostic AD, Gevers $D$, Sijander $H$, Vatanen $T$, Hyotylainen $T$, et al. (2015) The dynamics of the human infant gut microbiome in development and in progression toward type 1 diabetes. Cell Host Microbe 17: 260-273.

8. Sonnenburg ED, Smits $S$, Tikhonov $M$, Higginbottom S, Wingreen $\mathrm{N}$, et al. (2016) Diet-induced extinctions in the gut microbiota compound over generations. Nature 529: 212-215.

9. Espinoza J, Erez O, Romero R (2006) Preconceptional antibiotic treatment to prevent preterm birth in women with previous preterm delivery. Am J Obstrec Gynecol 194: 630-637.

10. Cardenas I, Mor G, Aldo P, Lang SM, Stabach P, et al. (2011) Placental viral infections sensitizes to endotoxin-induced preterm labor: a double hit hypothesis. Am J Repro Immunol 65:110-117.

11. Goldenberg RL, Hauth JC, Andrews WW (2000) Intrauterine infections and preterm delivery. N Engl J Med 342: 1500-1507.

12. Wang H, Hirsch E (2003) Bacterially-induced preterm labor and regulation of prostaglandin-metabolizing enzyme expression in mice: the role of Toll-like receptor 4. Biol Reprod 69: 1957-1963.

13. Agrawal V, Smart K, Jilling T, Hirsch E () Surfactant Protein (SPO-A suppresses preterm delivery and inflammation via TLR 2. PloS One 2-13; 8.e63990.

14. DiGiulio DB, Gervasi M, Romero R, Mazaki-Tovi S, Vaisbuch E, et al. (2010) Microbial invasion of the amniotic cavity in preeclampsia as assessed by cultivation and sequence-based methods. J Perinat Med 38: 503-513.

15. Racicot K, Cardenas I, Wunsche V, Aldo P, Guller S, et al. (2013) Viral infection of the pregnant cervix predisposes to ascending bacterial infection. J Immunol 191: 934-941.

16. Aagaard K, Ma J, Antony KM, Ganu R, Petrosino J, et al. (2014) The placenta harbors a unique microbiome. Sic Transl Med 6: 237 ra65.

17. Prince AL, Antony KM, Chu DM, Aagaard KM (2014) The microbiome, parturition, and timing of birth: more questions than answers. J Reprod Immunol 104: 12-19.

18. Adams Waldorf KM, Rubens CE, Gravett MG (2011) Use of nonhuman primate models to investigate mechanisms of infection-associated preterm birth. BJOG 118: 136-144.

19. Ramos BA, Kannien TT, Sisti G, Witkin SS (2015) Microorganisms in the female genital tract during pregnancy: Tolerance versus pathogenesis. Am J Reprod Immunol 73: 383-389.

20. Racicot K, Kwon JY, Aldo P, Abrahams V, El-Guindy A, et al. (2016) Type 1 interferon regulates the placental inflammatory response to bacteria and is targeted by virus: Mechanism of polymicrobial infection-induced preterm birth. Am J Reprod Immunol 75: 451-460.

21. AbrahaMsson TR, Jakobsson $H E$, Andersson AF, Bjorksten $B$, Engstrand L, et al. (2014) Low gut microbiota diversity in early 
infancy precedes asthma at school age. Clin Exp Allergy 44: 842-850.

22. Ismail IH, Boyle RJ, Licciardi PV, Oppedisano F, Lahtinen S, et al. (2016) Early gut colonization by Bifidobacterium breve and B. catenulatum differentially modulates eczema risk in children at high risk of developing allergic disease. Pediatr Allergy Immunol 8: 838-846.

23. Arrieta MC, Stiemsma LT, Dimitriu PA, Thorson L, Russel S, et al. (2015) Early infancy microbial and metabolic alterations affect risk of childhood asthma. Sci Transl Med 7: 307ra152.

24. Fijimura KE, Sitarik AR, Havstad S, Lin DL, Levan S, et al. (2016) Neonatal gut microbiota associates with childhood multisensitized atopy and T cell differentiation. Nat Med 22: 1187-1191.

25. Strauss JS, Pochi PE, Downing DT (1976) The sebaceous glands: twenty-five years of progress. J Invest Dermatol 67: 90-97.

26. Nicolaides N (1974) Skin lipids: their biochemical uniqueness. Science 186: 19-26.

27. Grice EA, Kong HH, Conlan S, Deming CB, Davis J, et al. (2009) Topographical and temporal diversity of the human skin microbiome. Science 324: 1190-1192.

28. Chu DM, Ma J, Prince AL, Antony KM, Seferovic MD, et al. (2017) Maturation of the infant microbiome community structure and function across multiple body sites and in relation to mode of delivery. Nat Med 23: 314-326.

29. Oh J, Conlan S, Polley EC, Segre JA, Kong HH (2012) Shifts in human skin and nares microbiota of healthy children and adults. Genome 4: 77.

30. Oh J, Byrd AL, Park M, Kong HH, Segre JA (2016) Temporal stability of the human skin microbiome. Cell 165: 854-866.

31. Chehoud C, Rafail S, Tyldsley AS, Seykora JT, Lambris JD, et al. (2013) Complement modulates the cutaneous microbiome and inflammatory milieu. Proc Natl Acad Sci 110: 15061-15066.

32. Ngy I, Pivarcsi A, Kis K, Koreck A, Bodai L, et al. (2006) Propionibacterium acnes and lipopolysaccharide induce the expression of antimicrobial peptides and proinflammatory cytokines/chemokines in human sebocytes. Microbes Infect 8: 2195-2205.

33. Cogen AL, Yamasaki K, Sanchez KM, Dorschner RA, Lai Y, et al. (2010) Selective antimicrobial action is provided by phenolsoluble modulins derived from Staphylococcus epidermidis, a normal resident of the skin. J Invest Dermatol 130: 192-200.

34. Nakatsuji T, Chen TH, Narala S, Chun KA, Two AM, et al. (2017) Antimicrobial from human skin commensal bacteria protect against Staphylococcus aureus and are deficient in atopic dermatitis. Sci Transl Med 9: eaah4680.

35. Chen EY, Fischbach AM, Beckaid Y (2018) Skin microbiota-host interactions. Nature 553: 427-436.

36. Fukuda $T$, Matsumura $T$, Ato $M$, Hamasaki $M$, Nishiuchi $Y$, et al. (2013) Critical roles for lipomannan and lipoarabinomannan in cell wall integrity of mycobacteria and pathogenesis of tuberculosis. MBio 4: e00472-12.

37. Bomar L, Brugger SD, Yost BH, Davies SS, Lemon KP (2016) Corynebacterium accolens releases antipneumococcal free fatty acids from human nostril and skin surface tracyglycerols. MBio 7: $1725-1740$
38. Linehan JL, Harrison OJ, Han SJ, Byrd AL, Vujkovic-Cvijin I, et al. (2018) Non-classical immunity controls microbiota impact on skin immunity and tissue repair. Cell 172: 784-796.

39. Caballero S, Pamer EG (2015) Microbiota-mediated inflammation and antimicrobial defense in the intestine. Annu Rev Immunol 33: 227-256.

40. Martin C, Burgel PR, Lepage P, Andrejak C, de Blic I, et al. (2015) Host-microbe interactions in distal airways: relevacnce to chronic airway diseases. Eur Respir Rev 24: 78-91.

41. Dickson RP, Erb-Downward JR, Martinez FJ, Huffnagle GB (2016) The microbiome and the respiratory tract. Annu Rev Physiol 78: 481-504.

42. Winslow CE (1908) A new method of enumerating bacteria in air. Science 28: 28-31.

43. Gleenson K, Eggli DF, Maxwell SL (1997) Quantitative aspiration during sleep in normal subjects. Chest 111: 1266-1272.

44. Huxley EJ, Viroslar J, Gray WR, Pierce AK (1978) Pharyngeal aspiration in normal adults and patients with depressed consciousness. Am J Med 64: 564-568.

45. Hilty M, Burke C, Pedro H, Cardens P, Bush A, et al. (2010) Disordered microbial communities in asthmatic airways. PLos One 5: e8578.

46. Morris A, Beck JM, Schloss PD, Campbell TB, Crotters K, et al. (2013) Comparison of the respiratory microbiome in healthy nonsmokers. Am J Respir Crit Care Med 187: 1067-1075.

47. Segal LN, Alekseyenko AV, Clemente JC, Kulkarni R, Wu B, et al. (2013) Enrichment of lung microbiome with a supraglottic taxa is associated with increased pulmonary inflammation. Microbiome 1: 19.

48. Erb-Downward JR, Thompson DL, Han MK, Freeman CM, McCloskey L, et al. (2011) Analysis of the lung microbiome in the "helathy" smoker and in COPD. PLos One 6: e16384.

49. Charlson ES, Diamond JM, Bittinger K, Fitzgerald AS, Yadav A, et al. (2012) Lung-enriched organisms and aberrant bacterial and fungal respiratory microbiota after lung transplant. Am J Respir Crit Care Med 186: 536-545.

50. Dominquez-Bello MG, Costello EK, Contreras M, Magris $M$,Hidalgo $G$, et al. (2010) Delivery mode shapes the acquisition and structure of the intail microbiota across multiple body habitats in newborns. Natl Acad Sci USA107: 11971-11975.

51. Igenito EP, Solway J, McFadden ER, Pichurko B, Bowman HF, et al. (1987) Indirect assessment of mucosal surface temperatures in the airways; theory and tests. J Appl Physiol 63: 2075-2083.

52. West JB (1978) Regional differences in the lung. Chest 74: 426-437.

53. Raghu G, Freudenberger TD, Yang B, Curtis JR, Spada C, et al. (2006) High prevalence of abnormal acid gastro-oesophageal reflux in idiopathic pulmonary fibrosis. Eur Respir J 27: 136-142.

54. Robinson NB, Dimango E (2014) Prevalence of gastroesophageal reflux in cystic fibrosis and implications for lung disease. Ann Am Thorac Soc 11: 964-968.

55. Ryerson CS, Cottin V, Brown KK, Collard HR (2015) Acute exacerbation of idiopathic pulmonary fibrosis: shifting the paradigm. Eur Respir J 46: 512-520.

56. Huang YJ, Nelson CE, Brodie EL, Desantis TZ, Raek MS, et al. (2011) Airway microbiota and bronchial hyper-responsiveness in 
patients with suboptimally controlled asthma. J Allergy Clin Immunol 127: 372-381.

57. Trompette A, Gollwitzer ES, Yadava K, Sichelstiel AK, Sprenger N, et al. (2014) Gut microbiota metabolism of dietary fibre influences allergic airway disease and hematopoiesis. Nat Med 20: 159-166.

58. Ley R, Backhed F, Turnbaugh P, Lozupone $C$, Knight $R$, et al. (2015) Obesity alters gut microbial ecology. Proc Natl Acad Sci 102: 11070-11075.

59. Sekirov I, Russel S, Antunes L, Finaly B (2010) Gut microbiota in health and disease. Physiol Rev 90: 859-904.

60. Fouhy F, Ross R, Fitzgerald G, Stanton C, Cotter P (2012) Composition of the early intestinal microbiota: knowledge, knowledge gaps and use of high-throughput sequencing to address these gaps. Gut Microbes 3: 203-220.

61. Adlerberth I, Wold A (2009) Establishment of the gust microbiota in western infants. Acta Paediatr 98: 229-238.

62. Tremaroli V, Backhed F (2012) Functional interactions between the gut microbiota and host metabolism. Nature 489: 242-249.

63. Qin J, Li Y, Cai Z, Li S, Zhu J, et al. (2012) A metagenomics-wide association study of gut microbiota in type 2 diabetes. Nature 490: 55-60.

64. Claesson M, Cusack S, Osullivan $\mathrm{O}$, Greene-Diniz R, de Weerd $\mathrm{H}$, et al. (2011) Composition, variability and temporal stability of the intestinal microbiota of the elderly. Proc Natl Acad Sci USA 108: 4586-4591.

65. Manichanh C, Borruel N, Casellas F, Guarner F (2012) The gut microbiota in IBD. Nat Rev Gastroenterol Hepatol 9: 599-608.

66. Sokol h, Pigneur B, Watterlot L, Lakhdari O, BermudezHUMARAN I, et al. (2008) Faecalibacterium prusnitzii is an antiinflammatory commensal bacterium identified by gut microbiota analysis of Crohn disease patients. Proc Natl Acad Sci USA 105: 16731-16736.

67. Morgan X, Tickle T, Sokol H, Gevers D, Devaney K, et al. (2012) Dysfunction of the intestine microbiome in inflammatory bowel disease and treatment. Genome Biol r79.

68. Arthur J, Perez-Chanona E, Muhlbauer M, Tomkovich S, Uronis J, et al. (2012) Intestinal inflammation targets cancer-inducing activity of the microbiota. Science 338: 120-123.

69. Kostic A, Gevers D, Pedamallu C, Michaud M, Duke F, et al. (2012) Genomic analysis identifies association of Fusobacterium with colorectal carcinoma. Genome Res 22: 292-298.

70. Strauss J, Kaplan G, Beck P, Rioux K, Panaccione R (2011) . Invasive potential of gut mucosa-derived Fusobacterium nucleatum positively correlates with IBD status of the host. Inflamm Bowel Dis 17: 1971-1978.

71. Brint E, Macsharry J, Fanning A, Shanahan F, Quigley E (2011) Differential expression of toll-like receptors in patients with irritable bowel syndrome. Am J Gastroenterol 106: 329-336.

72. Ghoshal U, Shukla R, Gwee K, Ng S, Quigley F (2012) The gut microbiota and irritable bowel syndrome: friend or foe? Int J Inflam 2012: 151085.

73. Rajilic-Stojanovic M, Biagi E, Heilig H, Kajander K, Kekkarein R, et al. (2011) Global and deep molecular analysis of microbiota signatures in fecal samples from patients with irritable bowel syndrome. Gastroenterology 141: 1792-1801.

74. Turnbaugh P, Ridaura V, Faith J, Rey F, Knight R, et al. (2009) The effect of diet on the human gut microbiome: a metagenomics analysis in humanized gnotobiotic mice. Sci transl Med 1: 6ra14.

75. Larsen N, Vogensen F, van den Berg F, Nielsen D, Andreasen A, et al. (2010) Gut microbiota in human adults with type 2 diabetes differs from non-diabeteic adults. Plos One 5: e9085. 